OPEN

SUBJECT AREAS:

NONLINEAR OPTICS

ULTRAFAST PHOTONICS

Received

5 February 2014

Accepted

10 July 2014

Published

20 October 2014

Correspondence and requests for materials should be addressed to

A.M.Z. (zheltikov@ physics.msu.ru)

\section{Time-domain spectroscopy in the mid-infrared}

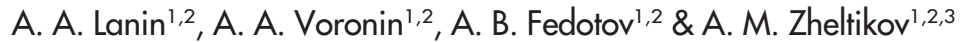

'Physics Department, International Laser Center, M.V. Lomonosov Moscow State University, Moscow 119992, Russia, ${ }^{2}$ Russian Quantum Center, ul. Novaya 100, Skolkovo, Moscow Region, 1430125 Russia, ${ }^{3}$ Department of Physics and Astronomy, Texas A\&M University, College Station TX 77843, USA.

When coupled to characteristic, fingerprint vibrational and rotational motions of molecules, an electromagnetic field with an appropriate frequency and waveform offers a highly sensitive, highly informative probe, enabling chemically specific studies on a broad class of systems in physics, chemistry, biology, geosciences, and medicine. The frequencies of these signature molecular modes, however, lie in a region where accurate spectroscopic measurements are extremely difficult because of the lack of efficient detectors and spectrometers. Here, we show that, with a combination of advanced ultrafast technologies and nonlinear-optical waveform characterization, time-domain techniques can be advantageously extended to the metrology of fundamental molecular motions in the mid-infrared. In our scheme, the spectral modulation of ultrashort mid-infrared pulses, induced by rovibrational motions of molecules, gives rise to interfering coherent dark waveforms in the time domain. These high-visibility interference patterns can be read out by cross-correlation frequency-resolved gating of the field in the visible generated through ultrabroadband four-wave mixing in a gas phase.

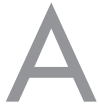

dvanced ultrafast laser technologies offer a variety of methods and tools to enhance resonant coupling between electromagnetic radiation and signature molecular modes, opening new avenues for ultrafast spectroscopy ${ }^{1}$, chemically specific microscopy ${ }^{2}$, quantum chemistry ${ }^{3}$, biomedical imaging ${ }^{4}$, and standoff detection $^{5}$. In the visible and near-infrared range, resonant frequency combinations ${ }^{6}$ and optimized pulse shapes ${ }^{7}$ can be used to enhance the coupling between the field and the molecular degrees of freedom through Raman-type processes, enabling time-resolved studies and quantum control of ultrafast processes in molecules ${ }^{7}$ and allowing chemically selective bioimaging ${ }^{6}$. In the terahertz range, where electromagnetic radiation can directly probe some of low-frequency characteristic molecular motions, time-domain spectroscopy of molecular bands proves to be advantageous for analytical applications and material characterization ${ }^{8}$.

The mid-infrared spectral range is unique, because electromagnetic radiation in this region can resonate with the most intense signature molecular bands. This drastically enhances the coupling between the field and molecular motions, thus offering numerous advantages for molecular spectroscopy $y^{9}$ and opening routes toward highly sensitive standoff detection ${ }^{5}$. Accurate measurements directly on a mid-infrared signal are, however, difficult because of the lack of efficient detectors and spectrometers for this spectral range. Here, we show that a combination of ultrashort pulses in the mid-infrared and nonlinear-optical methods of pulse characterization are ideally suited for the analysis of fundamental molecular motions, helping confront the long-standing challenges of chemically specific spectroscopy and recognition of molecular modes. We demonstrate that the spectral modulation of an ultrashort mid-infrared pulse induced by molecular rovibrational modes can give rise to highvisibility interference patterns and well-resolved echo revivals in the time domain, which can be read out by means of cross-correlation frequency-resolved optical gating based on broadband four-wave mixing in a gas phase, suggesting a powerful tool for the detection, recognition, and remote sensing of molecular vibrations and rotations.

Ultrashort waveforms with broadband coherent spectra in the mid-infrared play the central role in the timedomain technique for the detection and identification of molecular modes implemented in this work. When the central wavelength of such a waveform is tuned on resonance with a typical molecular rovibrational band, the mid-IR driver, due to its extremely large bandwidth, can interact with the entire manifold of rovibrational transitions, exciting a broadband rovibrational wave packet. As a part of this process, the energy is transferred from the mid-IR driver field to molecular motion, giving rise to narrowband absorption features in the spectrum of the mid-IR driver at the frequencies of individual molecular modes (Fig. 1a). In the time domain, these narrowband spectral dips translate into dark field waveforms (Fig. 1a), whose phase is shifted by $\pi$ relative to 


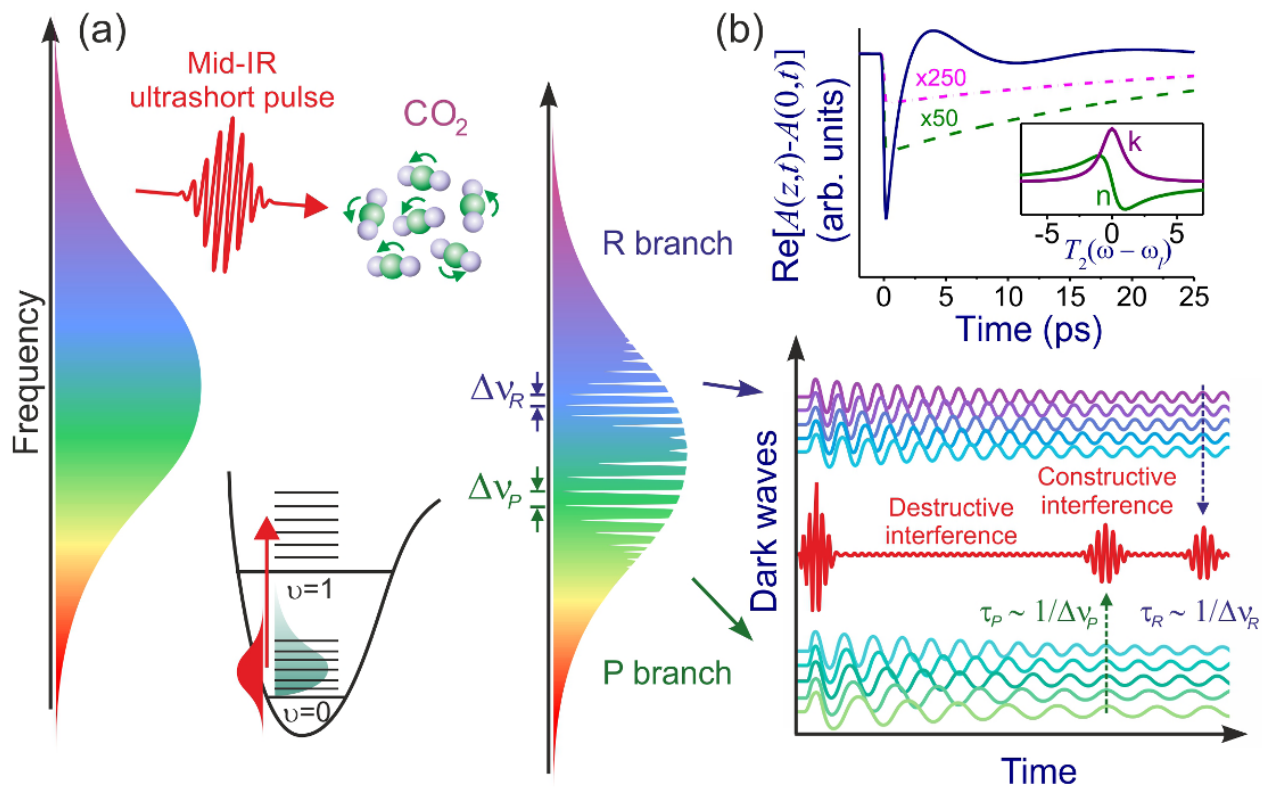

Figure $1 \mid$ (a) Time-domain spectral interferometry in the mid-infrared. When its central wavelength is tuned on resonance with a typical molecular rovibrational band, the mid-IR driver, due to its extremely large bandwidth, interacts with the entire manifold of rovibrational transitions, exciting a broadband rovibrational wave packet. As a part of this process, the energy is transferred from the mid-IR driver to molecular motion, giving rise to narrowband absorption features in the spectrum of mid-IR radiation at the frequencies of individual molecular modes, separated by spectral intervals $\Delta v_{\mathrm{P}}$ and $\Delta v_{\mathrm{R}}$ for the $\mathrm{P}$ and $\mathrm{R}$ rovibrational branches, respectively. In the time domain, these narrowband spectral dips translate into stretched dark field waveforms, as shown in the right panel. Due to the coherence preserved across the entire spectrum of the broadband mid-IR driver, these stretched pulses interfere with each other, giving rise to high-visibility fringes in the waveform of the transmitted mid-IR field (the right panel), with prominent echo field recurrences, observed at delay times $1 /\left(c \Delta v_{\mathrm{P}}\right)$ and $1 /\left(c \Delta v_{\mathrm{R}}\right)$ for the $\mathrm{P}$ and $\mathrm{R}$ rovibrational branches, respectively. Because the shape of these fringes is fully controlled by the spectrum of molecular rovibrational modes, all the information on a molecule encoded in absorption spectra can be retrieved from midIR pulse shapes, thus allowing molecular fingerprints to be read out through a careful analysis of mid-IR waveforms. (b) Dynamics of a dark wave: the envelope of a dark wave, $\operatorname{Re}[A(z, t)-A(0, t)]$, induced by interaction with a molecular transition with a Lorentzian lineshape with $T_{2}=30 \mathrm{ps}$ and $\Delta \omega_{l}=0$ for $\alpha_{l} z=0.1$ (pink line), 1 (green line), and 100 (navy line). The input laser profile has a Gaussian envelope and a pulse width of 160 fs. The spectral profiles of the real and imaginary parts $n$ and $\kappa$ of the complex refractive index $\bar{n}=n-i \kappa$ are shown in the inset.

the phase of the adjacent spectral components and whose duration is much longer than the duration of the incident ultrashort mid-IR driver (see the Methods section). Figure 1b illustrates the dynamics of one of such dark waveforms, produced by an idealized Lorentzian absorption line (see the Methods section for details). For short propagation paths, $\alpha_{l} z \ll 1$, with $\alpha_{l}$ being the absorption coefficient at the center of the line, the waveform has an exponentially decaying envelope (the dash--dotted line in Fig. 1b), as dictated by the Fourier transform of a Lorentzian spectrum. For longer propagation paths, however, propagation effects tend to distort this time-domain map of a molecular mode (see Fig. $1 \mathrm{~b}$ and the Methods section), generally making it more difficult to retrieve the parameters of molecular motions from this dark waveform.

Due to the coherence preserved across the entire spectrum of the broadband mid-IR driver, the dark waves produced by individual absorption lines in a molecular rovibrational band interfere with each other, giving rise to high-visibility fringes in the waveform of the transmitted mid-IR field (the right panel in Fig. 1a). Because the shape of these fringes is controlled by the spectrum of molecular rovibrational modes, the information on molecular modes encoded in the absorption spectra can be retrieved from mid-IR pulse shapes, thus allowing molecular fingerprints to be read out through a careful analysis of mid-IR waveforms with allowance for propagation effects (see Fig. $1 \mathrm{~b}$ and the Methods section). Such an analysis is, however, a challenging problem, because of the lack of efficient spectral analyzers and detectors for the mid-IR range. We show below in this paper that this challenge can be confronted using the four-wave mixing (FWM) of mid-IR pulses with well-characterized near-IR reference pulses in a gas phase. This process will be demonstrated to offer a powerful tool for ultrabroadband characterization of complex pulse shapes in the mid-IR, capable of recording ultrafast modulations of mid-IR waveforms, thus allowing unique barcodes of molecular modes to be accurately read out from mid-IR pulses.

In experiments, we use ultrashort mid-IR pulses produced through a difference-frequency mixing ${ }^{10,11}$ of the signal and idler fields delivered by the Ti: sapphire-laser-pumped optical parametric amplification of a broadband seed (Fig. 2a, see Ref. 12 and the Methods section for details). The central wavelength of broadband mid-IR fields delivered through this difference-frequency generation process was tunable from 2.7 to $15 \mu \mathrm{m}$. Energies above $0.5 \mu \mathrm{J}$ were delivered as the DFG output everywhere in the range of wavelengths from 2.7 to $13.6 \mu \mathrm{m}^{12}$. Pulse characterization of this DFG output was performed using cross-correlation frequency-resolved optical gating (XFROG) based on four-wave mixing (FWM) in a gas medium away from molecular resonances (see Ref. 12 and the Methods section). This technique, as shown in the earlier work ${ }^{13}$, enables dispersionfree measurements of pulse shapes and spectra of ultrashort mid-IR pulses and allows the spectral and temporal phases of such pulses to be retrieved in a robust and reliable way.

In our experiments, this method of time-domain spectroscopy in the mid-infrared was implemented by mixing mid-IR waveforms modulated by molecular motions with reference pulses in a gas medium through four-wave mixing and measuring the frequencyresolved FWM signal as a function of the delay time $\tau_{\mathrm{d}}$ between the mid-IR and reference pulses. The 50-fs, 808-810-nm output of the Ti: sapphire laser was used in our experiments as a source of 

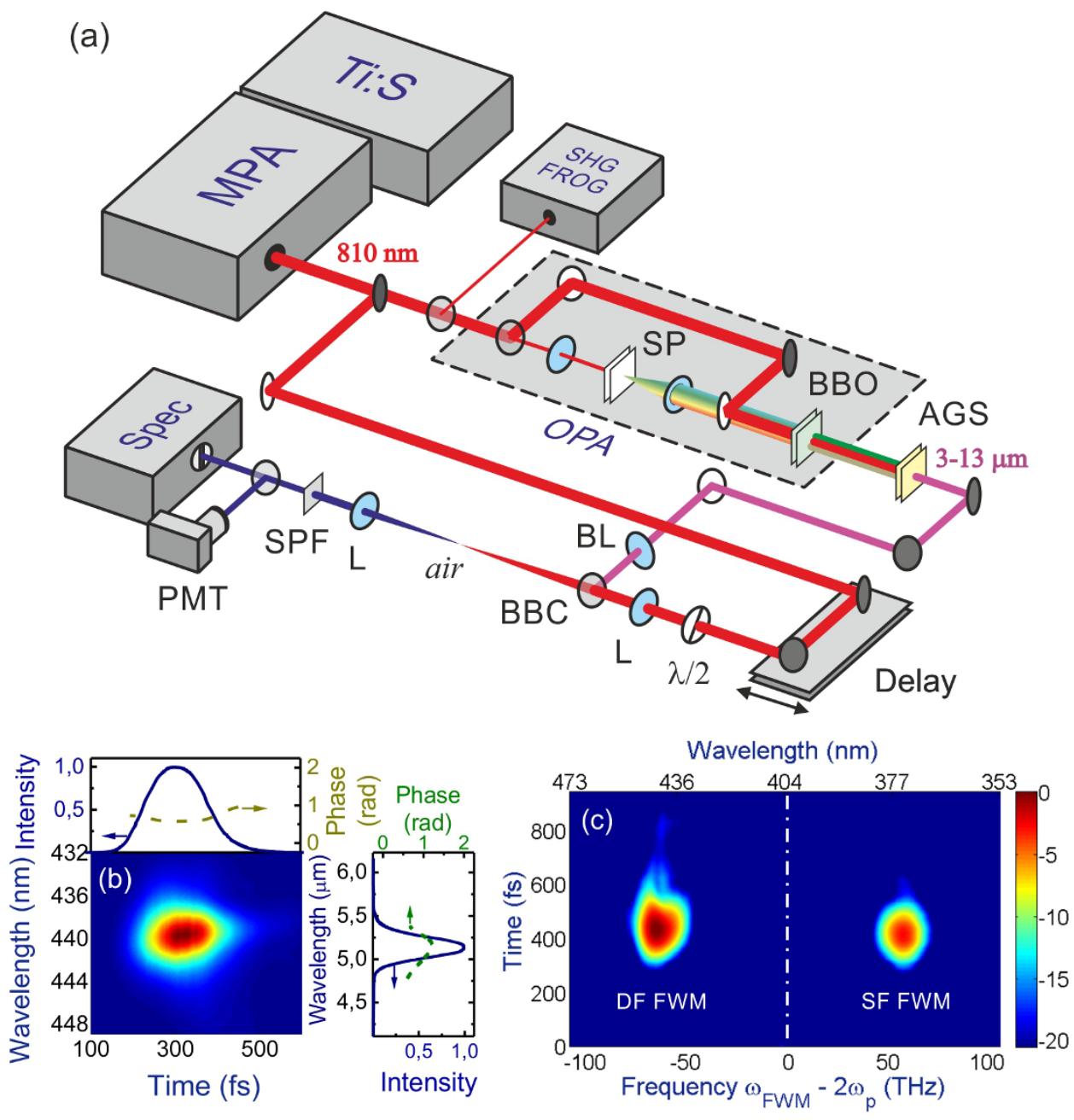

Figure $2 \mid$ (a) Diagram of the experimental setup: Ti: S, Ti: sapphire laser; MPA, multipass amplifier; OPA, optical parametric amplifier; AGS, AgGaS 2 crystal for difference-frequency generation; $\mathrm{SP}$, sapphire plate; $\mathrm{BBO}$, beta barium borate crystal, $\mathrm{BBC}, \mathrm{BaF}_{2}$ beam combiner; $\mathrm{BL}$, BaF ${ }_{2}$ lens; $\mathrm{L}$, $\mathrm{BK} 7$ glass lenses; Delay, tunable optical delay line, SPF, short-pass filter; PMT, photomultiplier tube; $\lambda / 2$, half-wave plate. (b) The FWM XFROG trace of the DFG output centered at $5.1 \mu \mathrm{m}$, measured with $\lambda_{\mathrm{p}}=810 \mathrm{~nm}$, shown along with its temporal envelope (solid curve in the panel above the trace), temporal phase (dashed curve in the panel above the trace), spectrum (solid curve in the panel to the right of the trace), and spectral phase (dashed curve in the panel right to the trace) retrieved from the XFROG trace. (e) Spectrochronograms of the sum- and difference-frequency FWM signals recorded with 50-fs 808$\mathrm{nm}$ Ti: sapphire pulses used as a pump. The $\lambda_{\mathrm{p}} / 2=404 \mathrm{~nm}$ axis is shown by the dashed line.

reference pulses, which were mixed with the mid-IR waveforms through the $\omega_{\mathrm{FWM}}=2 \omega_{\mathrm{p}} \pm \omega_{\mathrm{d}}$ FWM process, where the plus and minus signs correspond to sum- and difference-frequency FWM processes, respectively. In contrast to standard XFROG technology ${ }^{14}$, which is usually implemented using nonlinear processes in solidstate materials, XFROG based on FWM in a gas medium is intrinsically broadband, due to a weak dispersion of the gas medium, which helps avoid the stretching of pulses during XFROG measurements and which translates into broadband FWM phase matching, enabling characterization of ultrashort pulses in an ultrabroad spectral range. This version of XFROG is especially advantageous for ultrashort pulses in the mid-infrared as it naturally resolves the material loss problems, which frequently arise in this spectral range for solids. Pulse widths as short as 3.9 to 8 field cycles were detected using this FWM XFROG technique within the range of wavelengths from $3.2 \mu \mathrm{m}$ to $10.7 \mu \mathrm{m}$ (Fig. $2 \mathrm{~b}$ ), with the smallest pulse-width-tofield-cycle ratio of 3.9 achieved at $5.1 \mu \mathrm{m}$, where the DFG output pulse width was found to be $67 \mathrm{fs}^{12}$.

Typical FWM XFROG traces of mid-IR waveforms modulated by the rovibrational modes of carbon dioxide molecules and atmospheric water vapor are shown in Figs. 3a-3f. The difference between these traces and the XFROG traces of mid-IR pulses tuned off all the molecular resonances (Figs. 2b, 2c) is striking. Unlike off-resonance FWM XFROG traces, determined by an appropriate convolution of the mid-IR and reference pulses, the XFROG traces of mid-IR pulses tuned on a resonance with molecular modes exhibit well-resolved fringes along with a complex ring-down structure.

To understand the information encoded in the FWM XFROG traces of mid-IR pulses resonantly driving molecular motions, we need to examine the properties of quantum states of molecules manifested in their rovibrational motions. An antisymmetric stretch of linear molecules, such as carbon dioxide, for example, is coupled to rotations of the molecule, giving rise to characteristic absorption spectra (Fig. 3g), which consist of two distinctly different branches of rovibrational transitions with $\Delta J=+1$ (R branch) and $\Delta J=-1$ ( $\mathrm{P}$ branch) selection rules for the rotational quantum number ${ }^{15}$. Since rotations in rovibrational modes of molecules cannot be decoupled from vibrations, the separation between the individual rovibrational lines $\Delta v_{J}$ slightly varies with $J$ across each of the branches (Fig. 3i). However, the mean separation values for these branches can still be meaningfully defined as $\langle\Delta v\rangle=c\left(\Sigma_{J} \Delta v_{J} S_{J}\right) /\left(\Sigma_{J} S_{J}\right)$, where $S_{J}$ is the absorption line strength, giving, with the use of the reference data for 

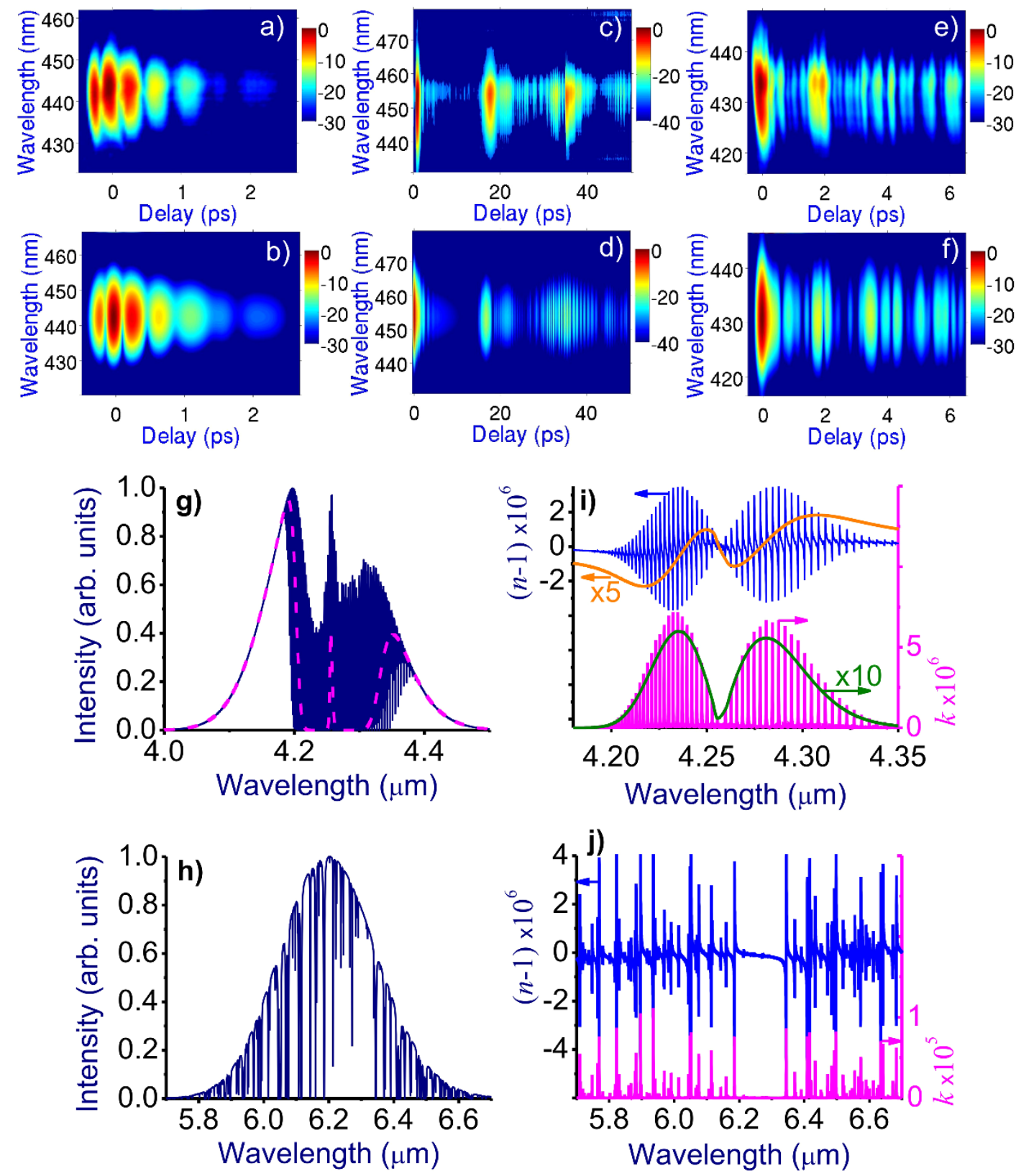

Figure 3 Cross-correlation frequency-resolved optical gating traces (a-f) of resonant mid-IR pulses with a central wavelength $\lambda_{0}$ equal to (a, b, $c$, $d$ ) $4.25 \mu \mathrm{m}$ and (e, f) $6.20 \mu \mathrm{m}$ and an input pulse width $\tau_{0}=160 \mathrm{fs}$ transmitted through $1.5 \mathrm{~m}$ of atmospheric air: (a, c, e) experiments and (b, d, f) simulations using Eqs.(1)-(6). The spectrum calculated using only the properly normalized envelopes of the $\mathrm{P}$ and $\mathrm{R}$ branches of the rotational spectrum of the antisymmetric stretch of a CO2 molecule is shown by the pink dashed line. (g, h) The spectra of resonant 160-fs mid-IR pulses with a central wavelength of (g) $4.25 \mu \mathrm{m}$ and (h) $6.20 \mu \mathrm{m}$ transmitted through $1.5 \mathrm{~m}$ of atmospheric air. (i) The spectral profiles of the absorption coefficient and refractive index of the antisymmetric stretch of a $\mathrm{CO} 2$ molecule calculated with the full model of the antisymmetric stretch band of CO2 (in pink and blue, respectively) and using only a properly normalized envelope of this band, without individual rovibrational lines (in green and orange). (j) The absorption coefficient (pink) and refractive index (blue) of water vapor calculated with the full model of the $\mathrm{H}-\mathrm{O}-\mathrm{H}$ bending mode of a water molecule.

a $\mathrm{CO}_{2}$ molecule $^{16},\langle\Delta v\rangle_{\mathrm{P}} \approx 1.82 \mathrm{~cm}^{-1}$ for the $\mathrm{P}$ branch and $<\Delta v>_{\mathrm{R}} \approx 1.29 \mathrm{~cm}^{-1}$ for the $\mathrm{R}$ branch.

The coherent waveforms corresponding to the missing, dark spectral components in the spectrum of the driver field, absorbed by $\mathrm{P}$ and R-branch molecular transitions, interfere constructively at delay times $\tau_{\mathrm{P} m, \mathrm{R} m}=m / c<\Delta v>_{P, R}$, with $m$ being an integer. This interference gives rise to prominent bright features in the XFROG traces of the mid-IR driver pulse, recurring at $\tau_{\mathrm{P} 1} \approx 18.3 \mathrm{ps}$ and $\tau_{\mathrm{R} 1} \approx$ 25.8 ps (Figs. 3c, 3d, 4a-4c), corresponding to molecular rovibrational transitions of the $\mathrm{P}$ and $\mathrm{R}$ branches, respectively. Similar to echo revival signals observed in the emission of rotating molecules, these high-intensity features clearly resolved in our XFROG traces originate from coherent ensembles of molecular rotators. However, unlike rotational echo revivals that show up in the radiative response of a molecular system, the coherent features in the FWM XFROG traces are synthesized through a constructive interference of dark mid-IR waveforms, sampled from the mid-IR driver by molecular motions (Fig. 1a). Notably, although this process can and should be described in terms of coherence induced in a quantum system, the evolution of mid-IR waveforms in our experiments is not reduced to free-induction decay ${ }^{17}$. Indeed, the laser fields continue to drive molecular modes along the entire propagation paths. The decay of 

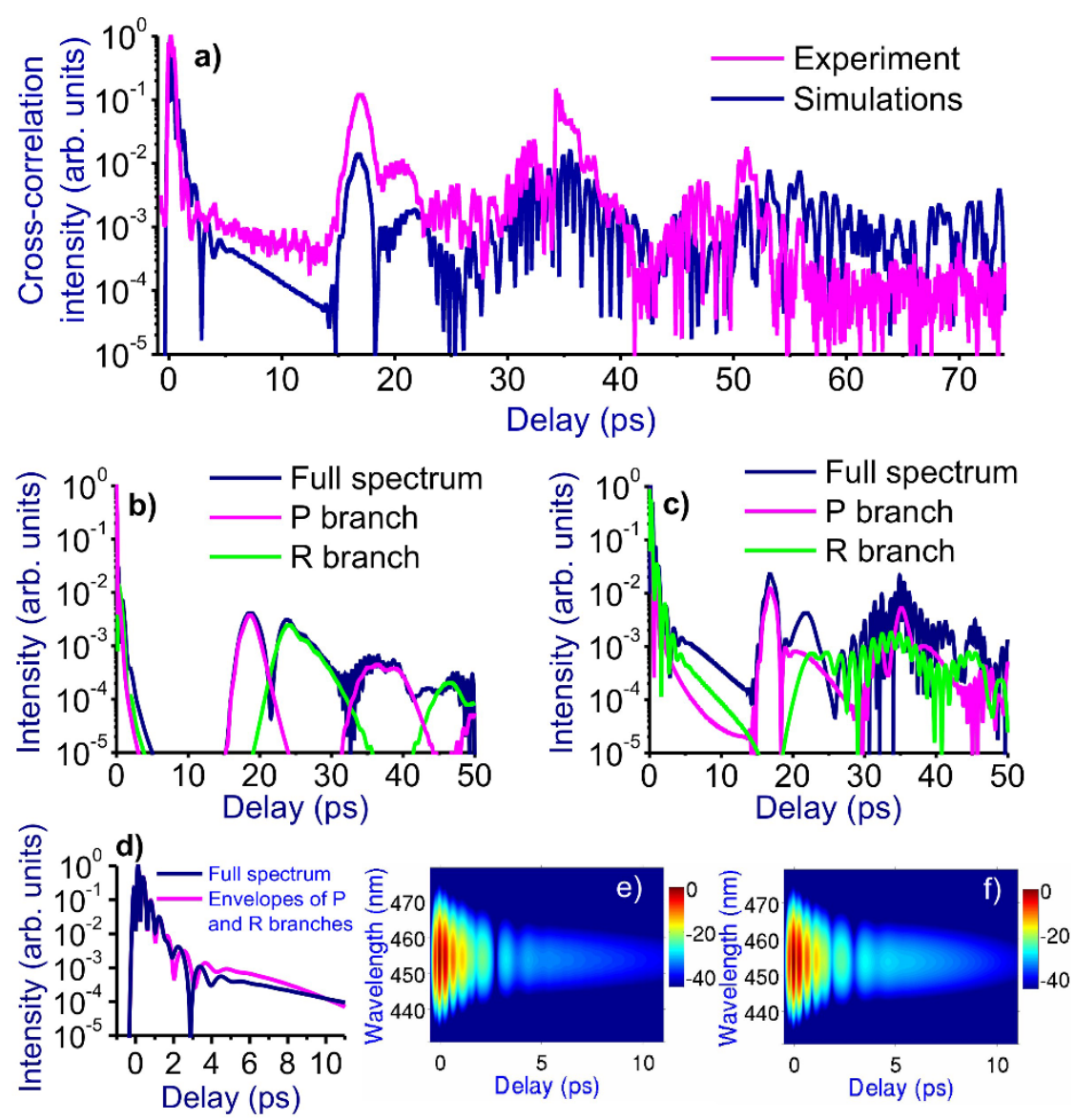

Figure $4 \mid$ (a) The cross-correlation trace of an ultrashort mid-IR pulse with $\tau_{0}=160$ fs and $\lambda_{0}=4.25 \mu \mathrm{m}$ transmitted through $1.5 \mathrm{~m}$ of atmospheric air: (pink line) experimental data, (blue line) simulations performed with the full model of the rotational spectrum of the antisymmetric stretch of a $\mathrm{CO}_{2}$ molecule. (b, c) Cross-correlation traces for a mid-IR pulse with the same input parameters transmitted through (b) $0.15 \mathrm{~m}$ and (c) $1.5 \mathrm{~m}$ of atmospheric air calculated using only the $\mathrm{P}$ (pink line) and $\mathrm{R}$ (green line) branches, as well as the full model (navy line) of the rotational spectrum of the antisymmetric stretch of a $\mathrm{CO}_{2}$ molecule. (d) The same as in panels (b, c) for a pulse transmitted through $1.5 \mathrm{~m}$ of atmospheric air calculated using (navy line) the full model of the rotational spectrum of the antisymmetric stretch of a $\mathrm{CO}_{2}$ molecule and (pink line) only the envelopes of the $\mathrm{P}$ and $\mathrm{R}$ branches of this spectrum without individual rotation lines. (e, f) XFROG traces for a mid-IR pulse with the same input parameters as above transmitted through $1.5 \mathrm{~m}$ of atmospheric air calculated using (e) the full model of the rotational spectrum of the antisymmetric stretch of a $\mathrm{CO}_{2}$ molecule and (f) only the envelopes of the $\mathrm{P}$ and $\mathrm{R}$ branches of this spectrum without individual rotation lines.

the induced coherence is therefore no longer free, giving rise to much more complicated field waveforms, as visualized by FWM XFROG traces. Simulations, performed for an ultrashort mid-IR pulse propagating in a medium with absorption and refraction described with a standard model ${ }^{15}$ of rovibrational bands of the antisymmetric stretch in linear molecules (see the Methods section), agree very well with the experimental results (Figs. 3c, 3d, 4a), confirming the assignment of all the key features in the XFROG and cross-correlation traces measured in our experiments.

Importantly, the coherent peaks induced in the XFROG and crosscorrelation traces of a mid-IR pulse by rovibrational molecular transitions are controlled by molecular rotational constants, thus providing a molecule-specific information. This is in contrast with the most intense feature always observed in XFROG and cross-correlation traces at zero delay time between the mid-IR and reference pulses (Fig. 3c, 3d, 4a-4c), which includes unresolved contributions due to all resonant and nonresonant coherent effects, suggesting no way of retrieving a molecule-specific information. Propagation effects, including absorption in the first place, tend to slightly shift the signature features in XFROG traces and lower their contrast. For our experimental conditions, as the propagation path is increased from 15 to $150 \mathrm{~cm}$ the first echo signal related to P-branch rovibrational transitions of $\mathrm{CO}_{2}$ is shifted from 18.3 to 16.7 ps, while its contrast decreases by two orders of magnitude (Figs. 4b, 4c). The first echo related to the $\mathrm{R}$ branch under the same conditions is shifted from 25.8 to 22.3 ps. Still, even with a $150-\mathrm{cm}$ propagation path, the ratio of the maximum intensity of the cross-correlation signal, corresponding to the peak of the P-branch echo signal, to the background intensity exceeds 100 (Figs. 3c, 3d, 4a-4c), making this echo signal the most intense and the most clearly resolved chemically specific feature in the optical response of the molecule.

Since a water molecule is an asymmetric top, its rotations do not give rise to clearly structured absorption bands. In our scheme of time-resolved measurements, this translates into a much less clearly pronounced structure of both spectra (Fig. 3h) and FWM XFROG traces (Fig. 3e) of ultrashort mid-IR pulses transmitted through the atmosphere within the $6.27-\mu \mathrm{m}$ absorption band of water vapor, corresponding to the $\mathrm{H}-\mathrm{O}-\mathrm{H}$ bending mode of a water molecule. Still, even though the analysis of the experimental data in this case is much more complicated, the experimental XFROG traces can be accurately reproduced (cf. Figs. $3 e$ and $3 \mathrm{f}$ ) with the use of the standard spectroscopic data (Fig. 3j). A complex manifold of intense individual rotational transitions observed in the spectrum of the absorption coefficient and refractive index of atmospheric water vapor (Fig. 3j) is seen to give rise to well-resolved bright features in the XFROG traces in Figs. 3e, encoding the information on molecular 
modes, which can be retrieved in certain regimes with an appropriate fitting of these traces (Figs. 3e, 3f).

In full agreement with basic Fourier-transform arguments, individual rovibrational molecular transitions constituting the $\mathrm{P}$ and $\mathrm{R}$ branches of $\mathrm{CO}_{2}$ absorption start to show up in XFROG and crosscorrelation traces only when the delay time $\tau_{\mathrm{d}}$ becomes comparable to $\tau_{\mathrm{P} 1}$ and $\tau_{\mathrm{R} 1}$ (Figs. $3 \mathrm{c}, 3 \mathrm{~d}, 4 \mathrm{a}-4 \mathrm{f}$ ). This argument helps identify important molecular signatures in cross-correlation and FWM XFROG traces. Specifically, individual rovibrational transitions of a $\mathrm{CO}_{2}$ molecule translate into a drastic, two-orders-of-magnitude increase in the FWM signal observed within the range of $\tau_{\mathrm{d}}$ from 15 to $17 \mathrm{ps}$, as well as into the second, slightly less pronounced rise of the FWM signal within the range of $\tau_{\mathrm{d}}$ from 18 to 21 ps (Fig. 4a). For shorter delay times, on the other hand, the properties of FWM XFROG traces are largely controlled by the overall shape of $\mathrm{P}$ and $\mathrm{R}$ rovibrational branches rather than the spectrum of individual rovibrational molecular transitions. This argument is verified by simulations where the full rovibrational spectrum of the antisymmetric stretch of $\mathrm{CO}_{2}$ (pink line in Fig. 3h) is replaced by properly normalized envelopes of the $\mathrm{P}$ and $\mathrm{R}$ branches (green line in Fig. $3 \mathrm{~h}$ ) without their individual spectral components corresponding to transitions with specific values of $J$. As can be seen from Figs. $4 d-4 f$, XFROG and cross-correlation traces calculated with the use of this approximation show almost no deviations from the results of calculations performed with the full model [Eqs. (1)-(6)] of rovibrational spectra of the antisymmetric stretch (Fig. 3i). This simplified model is seen to accurately reproduce and explain the most important features in the XFROG traces (Figs. 3a-3f) and spectra (Figs. 3g, 3h) observed in our experiments, offering a convenient tool to understand the key physical factors and tendencies in the evolution of ultrashort mid-IR pulses within molecular absorption bands.
The reconstruction of the spectrum of the complex refractive index of a molecule from FWM XFROG measurements is illustrated in Figs. 5a-5f and described in detail in the Methods section. Briefly, at the first step of this procedure, the pulse envelope and phase are retrieved from the FWM XFROG traces (Figs. 5a-5d). These retrieved data are then used to reconstruct the spectra of the absorption and refractive index of a molecule (Figs. 5d, 5f). The resolution of the retrieved spectra of the complex refractive index is determined by the total range of delay times in FWM XFROG measurements. Specifically, when the entire XFROG trace, measured with a delay time scan of 55 ps (Fig. 5a), is used, the full structure of the spectrum of the complex refractive index is retrieved with all its individual rovibrational lines (Fig. 5e) with a spectral resolution of about $0.6 \mathrm{~cm}^{-1}$. The envelopes of the absorption bands and the refractive index can be reconstructed (Fig. 5f), on the other hand, from the first few picoseconds of FWM XFROG traces (Fig. 5c).

Thus, the XFROG and cross-correlation traces of mid-IR pulses resonantly coupled to molecular rovibrational modes display signature, molecule-specific features, which show up on distinctly different time scales. These two classes of signatures characterize different aspects of quantized molecular motion. On the one hand, the well-resolved echo signals, appearing in the XFROG and crosscorrelation traces of mid-IR pulses on a 10-20-ps time scale provide quantitative information on molecules as quantum rotators. The fringes observed on the finer, subpicosecond time scale, on the other hand, are instrumental in understanding the content of ensembles of quantum molecular rotators and oscillators, allowing the temperature-dependent population distribution over molecular quantum states to be assessed.

To summarize, we have shown that a combination of ultrashort pulses in the mid-infrared and nonlinear-optical methods of pulse
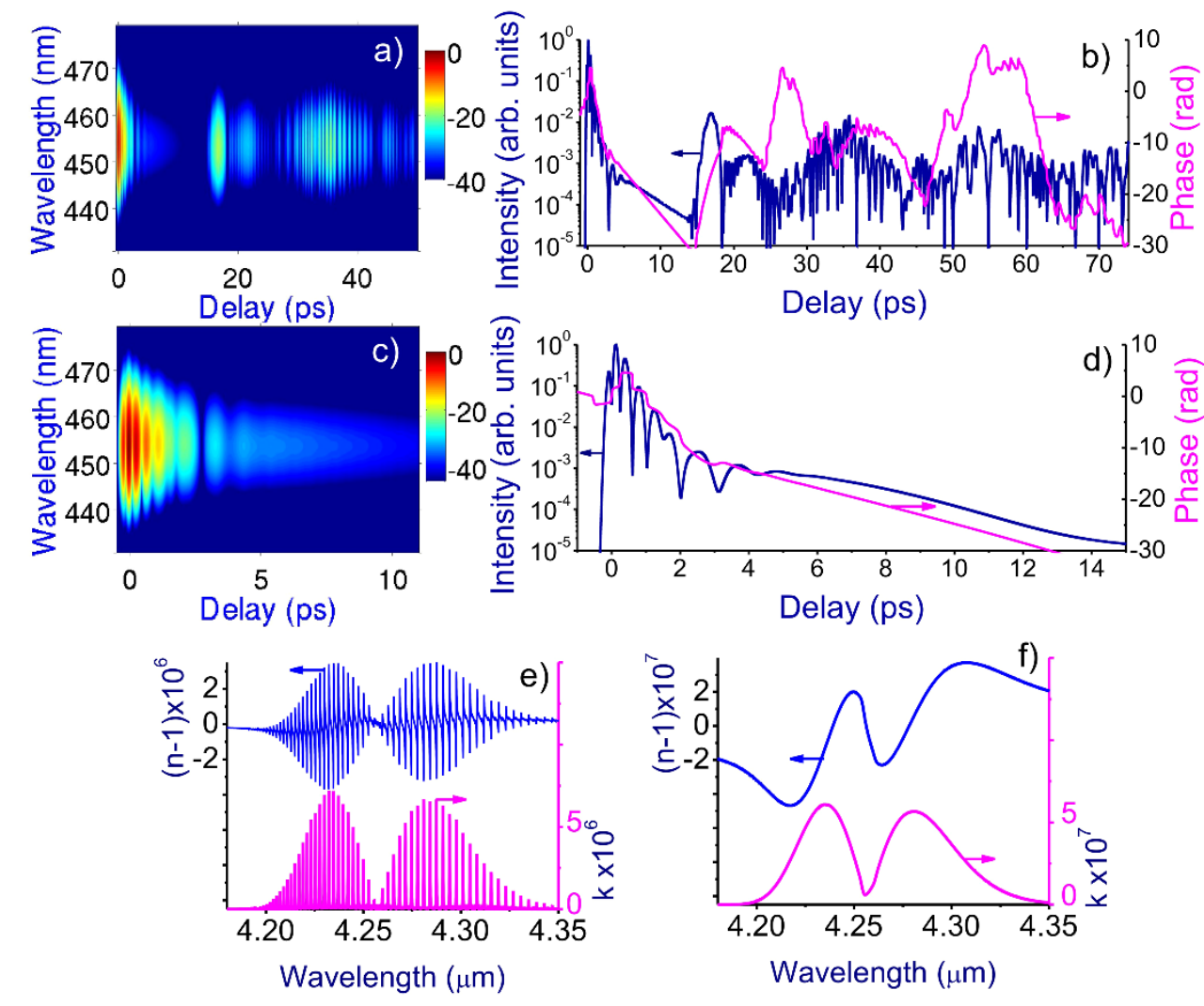

Figure $5 \mid$ Retrieving the spectroscopic data from FWM XFROG traces: (a) a typical FWM XFROG trace for a laser pulse with $\lambda_{0}=4.25 \mu \mathrm{m}$ and $\tau_{0}=$ 160 fs transmitted through $1.5 \mathrm{~m}$ of atmospheric air, (b) the pulse envelope and phase retrieved from the FWM XFROG trace, (c, d) close-up views of the first few picoseconds in the FWM XFROG trace (c) and the temporal envelope and phase profiles of the pulse (d), (e, f) the spectra of absorption (red line) and refractive index (blue line) of $\mathrm{CO}_{2}$ reconstructed from the FWM XFROG traces shown (e) in panel (a) and (f) in panel (c). 
characterization are ideally suited for the analysis of fundamental molecular motions, helping confront the long-standing challenges of chemically specific spectroscopy and recognition of molecular modes. We demonstrate that the spectral modulation of an ultrashort mid-infrared pulse induced by molecular rovibrational modes can give rise to high-visibility interference patterns and well-resolved echo revivals in the time domain, which can be read out by means of cross-correlation frequency-resolved optical gating based on broadband four-wave mixing in a gas phase, suggesting a powerful tool for the detection, recognition, and remote sensing of molecular vibrations and rotations. We have shown that our approach not only offers a tool to retrieve the absorption spectrum, which can be measured with a variety of techniques, most notably, FTIR spectroscopy, but allows the spectrum of the full complex refractive index to be reconstructed. A combination of the technique demonstrated here with chirped-pulse upconversion (CPU $)^{18,19}$ would be promising, as chirped pulses offer an elegant way of performing time-domain measurements without tunable delay lines. However, for the highest spectral resolution, as our study also shows, FWM XFROG measurements with a delay line tunable within a large range would still be needed, as the spectral resolution in CPU is limited by the pulse width of chirped pulses. Molecular spectroscopy with an unprecedented spectral resolution has been recently demonstrated using dual frequency combs ${ }^{20-22}$. This technique has been successfully employed within a broad range of wavelengths up to $2.4 \mu \mathrm{m}^{22}$, but still needs to be extended to the $4.0-6.2-\mu \mathrm{m}$ wavelength range, where experiments reported in this paper have been performed.

\section{Methods}

Generation of ultrashort pulses in the mid-infrared. Our method of ultrashortpulse generation in the mid-IR for the diagnostics of molecular motions involves two sequential stages of nonlinear-optical down conversion (Fig. 2a). At the first stage, 50 fs, $0.6-\mathrm{mJ}$ Ti: sapphire-laser pulses with a central wavelength of $800 \mathrm{~nm}$ are used to produce a broadband seed signal through supercontinuum generation in a sapphire plate and serve as a pump for an optical parametric amplification (OPA) of the seed signal in a BBO crystal. As a result of this OPA process $\omega_{\mathrm{p}}=\omega_{\mathrm{s}}+\omega_{\mathrm{i}}$, the Ti: sapphire pump field at the central frequency $\omega_{\mathrm{p}}$ amplifies the broadband seed signal at the frequency $\omega_{\mathrm{s}}$ and gives rise to an idler signal with a frequency $\omega_{\mathrm{i}}$. Adjusting phase matching through a rotation of the $\mathrm{BBO}$ crystal, we were able to tune the signal and idler wavelengths in the 1150-1580-nm and 1620-2300-nm ranges, respectively.

At the second stage of our mid-IR source (Fig. 2a), the signal and idler OPA outputs are used to generate a difference-frequency field through the DFG process $\omega_{\mathrm{d}}=\omega_{\mathrm{s}}-$ $\omega_{\mathrm{i}}$ in an $\mathrm{AgGaS}_{2}$ crystal $^{10,11}$. The central wavelength of the DFG signal generated as a result of this process can be tuned from 2.85 to $15 \mu \mathrm{m}$ by rotating the nonlinear crystal. The DFG radiation is separated from the signal and idler waves with a bandpass filter, blocking radiation with wavelengths shorter than $2.5 \mu \mathrm{m}$. The spectrum of the DFG radiation is measured with the use of a cooled $\mathrm{HgCdTe}$ detector and a home-made monochromator with replaceable gratings. In the monochromator, 300-grooves $/ \mathrm{mm}$ gratings, used to analyze the spectrum of radiation with wavelengths shorter than $4 \mu \mathrm{m}$, were replaced by 150 -grooves/mm gratings for measurements in the longerwavelength region. The power of the DFG output was measured using a thermal power meter. Energies above $0.5 \mu \mathrm{J}$ were achieved for the short-pulse DFG output everywhere in the range of wavelengths from 2.7 to $13.6 \mu \mathrm{m}^{12}$.

Time- and frequency-resolved measurements with ultrashort pulses in the midinfrared. Characterization of ultrashort pulses in the mid-infrared was performed by using cross-correlation frequency-resolved optical gating (XFROG) based on fourwave mixing (FWM) in a gas medium ${ }^{12,13}$. To this end, a mid-IR pulse is combined with a reference Ti: sapphire laser pulse on a $0.6-\mathrm{mm}$-thick $\mathrm{BaF}_{2}$ plate and is focused into a gas medium with a $25-\mathrm{cm}$-focal-length $\mathrm{BaF}_{2}$ lens (Fig. 2a). The FWM signal produced in this geometry is collimated with a $7.5-\mathrm{cm}$-focal-length $\mathrm{BK} 7$ glass lens, separated from the infrared beams with a set of appropriate filters, and analyzed using a Hamamatsu H9307-02 photomultiplier tube and an Ocean Optics spectrometer. The third harmonic of the Ti: sapphire laser pump is efficiently suppressed due to absorption in BK7 glass. The signal and idler pulses delivered by the OPA are characterized using the XFROG technique by sum-frequency-mixing these pulses with the 47-fs, $810-\mathrm{nm}$ Ti: sapphire-laser output in a thin, 50- $\mu \mathrm{m}$ BBO crystal.

Both the sum- and difference-frequency FWM processes are observed in this experimental scheme. The shapes of XFROG traces of the $\omega_{\mathrm{FWM}}=2 \omega_{\mathrm{p}}+\omega_{\mathrm{d}}$ and $\omega_{\mathrm{FWM}}=2 \omega_{\mathrm{p}}-\omega_{\mathrm{d}}$ FWM signals recorded for the same mid-IR pulse with $\lambda_{\mathrm{d}} \approx$ $4.8 \mu \mathrm{m}$ are shown in Fig. 2e. Since both FWM processes involve the same input optical fields, the shapes of these traces are symmetric with respect to the $\lambda_{\mathrm{p}}=$ $404 \mathrm{~nm}$ axis (the dashed line in Fig. 2c). The information on the mid-IR pulse encoded in XFROG traces produced through sum- and difference-frequency FWM is thus fully equivalent. However, due to a favorable phase matching, the difference- frequency FWM process $\omega_{\mathrm{FWM}}=2 \omega_{\mathrm{p}}-\omega_{\mathrm{d}} \mu \mathrm{m}$, as can be seen from Fig. $2 \mathrm{c}$, is much more efficient than its sum-frequency counterpart and is therefore much better suited for the characterization of ultrashort mid-IR pulses. It is this difference-frequency branch of FWM that is used for XFROG measurements on the DFG output (Figs. 2b, 2c) and mid-IR pulses transmitted through the atmospheric air within molecular absorption bands (Figs. 3a-3f).

Characterization of mid-infrared ultrashort pulses at the output of the DFG source. A typical FWM XFROG trace of the DFG output with a central wavelength of $5.1 \mu \mathrm{m}$ is presented in Fig. $2 \mathrm{~b}$. A pulse shape retrieved from this trace, shown by the solid line in the panel above the trace, has a pulse width of $155 \mathrm{fs}$ with a moderate chirp (dashed line in the panel above Fig. 2b). The spectrum of this pulse (the panel to the right of Fig. 2c) supports a transform-limited pulse shorter than $70 \mathrm{fs}$. Indeed, with a careful adjustment of beam focusing, as well as positioning of the OPA and DFG crystals relative to the beam waists for optimal chirp precompensation in the DFG process, pulses with a pulse width of $67 \mathrm{fs}$ were generated at $5.1 \mu \mathrm{m}$ with a pulse energy of $2.8 \mu \mathrm{J}$ right at the output of the DFG crystal $^{12}$, corresponding to only 3.9 field cycles under the pulse envelope. Sub-five-cycle mid-IR pulses were also produced in our experiments within the range from 6.2 to $6.7 \mu \mathrm{m}$, i.e., in the atmospheric water absorption band. In the atmospheric-window mid-IR range, subfive-cycle 145 -fs pulses with a pulse energy of $1.4 \mu \mathrm{J}$ have been produced at $\lambda_{\mathrm{d}} \approx$ $9.5 \mu \mathrm{m}$. On the opposite side of the DFG output tunability range, that is, in the 7-14$\mu \mathrm{m}$ atmospheric-window region, pulses as short as $308 \mathrm{fs}$ were delivered at a central wavelength of $10.7 \mu \mathrm{m}$. These pulses were compressible to $244 \mathrm{fs}$ through ideal chirp compensation to a transform-limited waveform, with their spectra stretching at the 0.3 level from 10.3 to $11 \mu \mathrm{m}$

Rovibrational spectra of the antisymmetric stretch in linear molecules. The optical response of rovibrational wave packets of molecules was simulated using a standard model ${ }^{15}$ of a linear molecule with an absorption spectrum consisting of $\mathrm{R}$ and $\mathrm{P}$ branches, corresponding to selection rules $\Delta J=+1$ and $\Delta J=-1$, respectively, where $J$ is the rotational quantum number. The frequencies of rovibrational transitions within these branches are given by

$$
\begin{aligned}
& v_{R}=v_{1}(J+1)-v_{0}(J) \\
& v_{P}=v_{1}(J-1)-v_{0}(J)
\end{aligned}
$$

with the frequencies $v_{v}$ of the lower $(v=0)$ and upper $(v=1)$ states involved in rovibrational transitions (see Fig. 1a) calculated with an approximation

$$
\begin{aligned}
& v_{0}(J) \approx G_{0}+B_{0} J(J+1), \\
& v_{1}(J) \approx G_{1}+B_{1} J(J+1),
\end{aligned}
$$

where $G_{v}$ are the vibrational terms and $B_{v}$ are the rotational constants of a molecule. The intensities of individual spectral lines within the $\mathrm{R}$ and $\mathrm{P}$ branches of the rovibrational absorption band are given by ${ }^{15}$

$S_{R, P}(J, T)=\frac{v_{R, P}}{\tilde{v}_{0}} S_{0}(T) L_{R, P}(J)\left[\exp \left(-h v_{0}(J) / k T\right) / Q(T)\right]\left[1-\exp \left(-h v_{R, P} / k T\right)\right]$,

where $T$ is the temperature, $\tilde{v}_{0}=G_{1}-G_{0}$ is the frequency of purely vibrational transition, $S_{0}(T)$ is the rotationless line strength, $L(J)$ is the Honl-London factor, $Q(T)$ $=\Sigma_{J}(2 J+1) \exp \left\{-h v_{0}(J) / k T\right\}$ is the rotational partition sum, $k$ is the Boltzmann constant, and $h$ is the Planck constant.

With the absorption spectrum $S(\omega)$ determined from Eq. (5), the refractive index $n(\omega)$ is calculated through the Kramers-Kronig transform of $S(\omega)$.

The spectrum of a mid-IR pulse is then found as

$$
A(z, \omega)=A(z=0, \omega) \exp \left(\mathrm{i} k^{\prime} z\right)
$$

where $k^{\prime}$ includes the complex refractive index and $z$ is the propagation coordinate. The waveform of this pulse is calculated by taking the Fourier transform of Eq. (6).

For the asymmetric stretch of $\mathrm{CO}_{2}$ molecules, we take ${ }^{16} G_{0}=0, G_{1}=$ $2349.14291 \mathrm{~cm}^{-1}, B_{0}=0.39021889 \mathrm{~cm}^{-1}, B_{1}=0.38714135 \mathrm{~cm}^{-1}, L_{R}(J)=J+1$, $L_{P}(J)=J$, and $S_{0}(T=296 \mathrm{~K})=916076 \cdot 10^{-22} \mathrm{~cm} / \mathrm{mol}$. The rovibrational absorption spectrum calculated with these parameters is shown by the pink line in Fig. 3i. The refractive index calculated through the Kramers-Kronig transform of this absorption spectrum is presented by the blue line in the same figure. Calculations performed with the envelope of the absorption spectrum and the corresponding spectral profile of the refractive index (shown by the green and orange lines in Fig. 3i) are seen to reproduce the key features in cross-correlation (Fig. 4d) and XFROG (Figs. 4e, 4f) traces for delay times shorter than approximately $10 \mathrm{ps}$. For longer delay times, features related to individual rovibrational molecular transitions start to show up in the XFROG traces (see Figs. 3c, 3d, 4a)

Dark wave evolution. For an isolated Lorentzian absorption line with a half-width $1 /$ $T_{2}$ and peak absorption $\alpha_{l}$ at the central frequency $\omega_{l}$, the solution to Eq. (6) is written $\mathrm{as}^{23}$ 


$$
A(z, t)=A(0, t)-\int_{-\infty}^{t} R_{l}\left(t-t^{\prime}\right) A\left(0, t^{\prime}\right) d t^{\prime}
$$

where $A(0, t)$ is the envelope of the input pulse,

$R_{l}(z, t)=\varepsilon_{l} \exp \left\{-\left(T_{2}^{-1}-i \Delta \omega_{l}\right) t\right\} J_{1}\left(\sqrt{2 \varepsilon_{l} t}\right) / \sqrt{2 \varepsilon_{l} t}, \varepsilon_{l}=\alpha_{l} z / T_{2}, \Delta \omega_{l}=\omega_{0}-\omega_{l}$, and $\omega_{0}$ is the central frequency of the laser pulse.

The minus sign in front of the second term on the right-hand side of Eq. (7) reflects a $\pi$ phase shift of the dark wave induced by an absorption line relative to the input field.

In the approximation $\alpha_{l} z \ll 1$, pump depletion can be neglected, and Eq. (7) is reduced to an exponential dark wave (the pink line in Fig. 1b):

$$
A(z, t) \approx A(0, t)-\frac{1}{2} \varepsilon_{l} \Theta(t) \exp \left\{-\left(T_{2}^{-1}-i \Delta \omega_{l}\right) t\right\} \int_{-\infty}^{\infty} A(0, t) d t,
$$

where $\Theta(t)$ is Heaviside step function.

In this approximation, time-domain evolution of a broadband field waveform within a molecular absorption band can be understood in terms of the interference of exponential dark waves, as described by the second term on the right-hand side of Eq. (8). In the regime $\alpha_{l} z \gg 1$, however, pump depletion within absorption lines distorts this simple picture of dark-wave interference (Fig. 1b). In this case, the dynamics of an ultrashort mid-IR pulse within a molecular absorption band is analyzed through a straightforward Fourier transform of Eq. (6).

Retrieval of the complex refractive index. The spectrum of the complex refractive index of a molecule is retrieved from FWM XFROG traces through a two-step procedure (Figs. 5a-5f). At the first step, the pulse envelope $A(z, t)$ and its phase are retrieved from the FWM XFROG traces (Figs. 5a-5d). At the second step (Figs. 5e, 5f), Eq. (7) is used to reconstruct the spectra of the refractive index, $n(\omega)$, and absorption, $\kappa(\omega)$, from the pulse envelope and its phase retrieved from FWM XFROG traces at the first step, as the real and imaginary parts of the complex refractive index, appearing in Eq. (7):

$$
\begin{aligned}
\kappa(\omega) & =\operatorname{Re}\left[\frac{c}{\omega z} \ln \left(\frac{A(z=0, \omega)}{A(z, \omega)}\right)\right], \\
n(\omega) & =\operatorname{Im}\left[\frac{c}{\omega z} \ln \left(\frac{A(z=0, \omega)}{A(z, \omega)}\right)\right],
\end{aligned}
$$

where $A(z, \omega)$ is the Fourier transform of $A(z, t)$.

1. Zewail, A. H. Laser Femtochemistry. Science 242, 1645-1653 (1988)

2. Saar, B. G. et al. Video-Rate Molecular Imaging in Vivo with Stimulated Raman Scattering. Science 330, 1368-1370 (2010).

3. Warren, W. W., Rabitz, H. \& Dahleh, M. Coherent Control of Quantum Dynamics: The Dream Is Alive. Science 259, 1581-1589 (1993).

4. Freudiger, C. W. et al. Label-Free Biomedical Imaging with High sensitivity by Stimulated Raman Scattering Microscopy. Science 322, 1857-1860 (2008).

5. Malevich, P. N. et al. Ultrafast-laser-induced backward stimulated Raman scattering for tracing atmospheric gases. Opt. Express 20, 18784-18794 (2012).

6. Dudovich, N., Oron, D. \& Silberberg, Y. Single-pulse coherently controlled nonlinear Raman spectroscopy and microscopy. Nature 418, 512-514 (2002).

7. Herek, J. L., Wohlleben, W., Cogdell, R. J., Zeidler, D. \& Motzkus, M. Quantum control of energy flow in light harvesting. Nature 417, 533-535 (2002).

8. Tonouchi, M. Cutting-edge terahertz technology. Nature Photonics 1, 97-105 (2007).

9. Huth, F., Schnell, M., Wittborn, J., Ocelic, N. \& Hillenbrand, R. Infraredspectroscopic nanoimaging with a thermal source. Nature Mater. 10, 352-356 (2011).

10. Petrov, V., Rotermund, F. \& Noack, F. Generation of high-power femtosecond light pulses at $1 \mathrm{kHz}$ in the mid-infrared spectral range between 3 and $12 \mu \mathrm{m}$ by second-order nonlinear processes in optical crystals. J. Opt. A: Pure Appl. Opt. 3, R1 (2001).

11. Kaindl, R. A. et al. Generation, shaping, and characterization of intense femtosecond pulses tunable from 3 to $20 \mu \mathrm{m}$. J. Opt. Soc. Am. B 17, 2086-2094 (2000).

12. Lanin, A. A., Fedotov, A. B. \& Zheltikov, A. M. Ultrabroadband XFROG of fewcycle mid-infrared pulses by four-wave mixing in a gas. J. Opt. Soc. Am. B 31, 1901-1905; doi:10.1364/JOSAB.31.001901 (2014).

13. Nomura, Y. et al. Phase-stable sub-cycle mid-infrared conical emission from filamentation in gases. Opt. Express 20, 24741-24747 (2012).

14. Reid, D. T., Loza-Alvarez, P., Brown, C. T. A., Beddard, T. \& Sibbett, W. Amplitude and phase measurement of mid-infrared femtosecond pulses by using cross-correlation frequency-resolved optical gating. Opt. Lett. 25, 1478-1480 (2000).

15. Herzberg, G. Molecular spectra and molecular structure: III. Electronic spectra and electronic structure of polyatomic molecules (Van Nostrand, Princeton, NJ, 1966).

16. Rothman, L. S., Hawkins, R. L., Watson, R. B. \& Gamache, R. R. Energy levels, intensities, and linewidths of atmospheric carbon dioxide bands. J. Quant. Spectrosc. Radiat. Transfer 48, 537-551 (1992)

17. Coddington, I., Swann, W. C. \& Newbury, N. R. Time-domain spectroscopy of molecular free-induction decay in the infrared. Opt. Lett. 35, 1395-1397 (2010)

18. Nomura, Y. et al. Single-shot detection of mid-infrared spectra by chirped-pulse upconversion with four-wave difference frequency generation in gases. Opt Express 21, 18249-18257 (2013).

19. Nee, M. J., McCanne, R., Kubarych, K. J. \& Joffre, M. Two-dimensional infrared spectroscopy detected by chirped pulse upconversion. Opt. Lett. 32, 713-715 (2007).

20. Coddington, I., Swann, W. C. \& Newbury, N. R. Coherent Multiheterodyne Spectroscopy Using Stabilized Optical Frequency Combs. Phys. Rev. Lett. 100, 013902 (2008).

21. Bernhardt, B. et al. Cavity-enhanced dual-comb spectroscopy. Nature Photon. 4, 55-57 (2010)

22. Bernhardt, B. et al. Mid-infrared dual-comb spectroscopy with $2.4 \mu \mathrm{m} \mathrm{Cr}^{2+}: \mathrm{ZnSe}$ femtosecond lasers. Appl. Phys. B 100, 3-8 (2010).

23. Laubereau, A. \& Kaiser, W. Vibrational dynamics of liquids and solids investigated by picosecond light pulses. Rev. Mod. Phys. 50, 607-665 (1978).

\section{Acknowledgments}

This research was supported in part by the Russian Foundation for Basic Research (project nos.13-02-01465, 13-02-92115, and 14-02-00784) and the Welch Foundation (Grant No. A-1801). Development of the mid-infrared source of ultrashort pulses and spectroscopic studies in the mid-infrared were supported by the Russian Science Foundation (project no. 14-12-00772). The research of A.A.V. is also supported by the Dynasty Foundation.

\section{Author contributions}

A.A.L. designed and performed the experiments, analyzed the data, and prepared the graphic material. A.A.V. analyzed the data and prepared the graphic material. A.B.F. designed the experiments and participated in discussions. A.M.Z. designed and oversaw the experiments, analyzed the data, and wrote the paper.

\section{Additional information}

Competing financial interests: The authors declare no competing financial interests.

How to cite this article: Lanin, A.A., Voronin, A.A., Fedotov, A.B. \& Zheltikov, A.M. Time-domain spectroscopy in the mid-infrared. Sci. Rep. 4, 6670; DOI:10.1038/srep06670 (2014)

This work is licensed under a Creative Commons Attribution-NonCommercialShareAlike 4.0 International License. The images or other third party material in this article are included in the article's Creative Commons license, unless indicated otherwise in the credit line; if the material is not included under the Creative Commons license, users will need to obtain permission from the license holder in order to reproduce the material. To view a copy of this license, visit http:// creativecommons.org/licenses/by-nc-sa/4.0/ 